\title{
UAV Ad Hoc Network System Design
}

\author{
Yongjian Luo ${ }^{\mathrm{a}}$, Xugang Liub ${ }^{\mathrm{b}}$, Ruifeng $\mathrm{Yu}^{\mathrm{c}}$, Jin Mengc, Ran $\mathrm{Li}^{\mathrm{c}}$ \\ Xi' an Communication Institute, Xi'an 710106, China \\ a 51375908@qq.com, ${ }^{\mathrm{b}}$ 813086903@qq.com, ${ }^{\mathrm{c}}$ 23414388@qq.com
}

Keywords: UAV; Ad hoc network; network structure; networking technology

\begin{abstract}
According to the special application of UAV network and its requirement of flexible and dynamic networking, this paper proposes a UAV network structure design based on ad hoc network. This structure provides a whole connected networking for UAV network, including the design of key technologies such as system structure, networking technology and routing protocol, so that the requirements of UAV quickly moving self-organizing network and frequently changing topology can be satisfied.
\end{abstract}

\section{Introduction}

In recent years, modern wars have shown a clear trend of unmanned development. UAVs have many advantages in modern warfare. They are featured as low cost, good concealment, strong vitality, ease of casualty and easy replenishment, fast taking-off and landing, flexible operation and diverse-suitability for multiple task types. In order to meet the needs of future warfare, UAVs are moving from multiple support functions, such as simple auxiliary training and reconnaissance and surveillance, to multi-functional directions such as communications relay, battlefield management, fire guidance, damage assessment, early warning, electronic warfare, attack and killing. UAVs can gradually satisfy the needs of low and high altitude coverage, long stay in war zone, miniaturization, multi-function, high performance, working in clusters. And its significant combat effectiveness, low damage cost, easy to equip gives it a pivotal role in war combat. The premise of multi-machine collaborative combat is to achieve independent measurement and control communications integration in unmanned aircraft groups, that is, UAV network with strong communication capabilities, information perception and destruction abilities should be equipped.

\section{Structure Design}

Compared with the traditional Ad hoc network, UAV has a broader network coverage, faster moving speed, which result in frequent changes in the network topology. At the same time, UAVs usually need to communicate with each other, cooperatively perform tasks with strong group mobility, which sets higher requirements for network communication.

Hierarchical design, which divides the entire communication protocol into a number of relatively independent sub-layer, is often used in network communication protocol design. One of the keys to the success of Internet technology stems from the adoption of a layered communications protocol structure.

Each communication drone supports a complete communication protocol stack structure, including the complete communication protocol from the application layer to the physical layer. In view good scalability and robustness of TCP / IP protocol family, we intend to use the TCP / IP protocol stack as the target network protocol stack frame as TCP / IP is not designed for wireless communication networks, and its basic design philosophy is based on Strict hierarchical design. So TCP / IP must be based on its existing framework to expand, making it suitable for the target research network environment. 


\section{Networking technology}

So far, most UAVs in the world commit only a single mission at a time. When use UAVs, they usually control them directly through the ground control center, thus forming a ray star structure model starting from a command center of the ground station to multiple UAVs in the air. According to the report, the main modes of communication used by UAVs in the United States are still based on the ground control center. The ground control center control drone programs are:

1) Direct remote control of one or several UAVs, which is commonly used, but limited by the curvature of the ground and the limited distance traveled by UAVs;

2) Use a number of UAVs as indirect relays and then achieve the control of the remote UAV and information transmission. The scope of UAV communication can be largely expanded by forming UAV network and multi-hop communication.

UAV group flight is based on a certain rule so that the UAV can maintain a certain distance to avoid collision with each other. The rules should focus on the formation of a number of UAV formation so that the data link between the ground station and multiple UAVs and the control and management of multiple UAVs by the ground station have become the focuses of our research.

\section{Routing Protocol}

Ad hoc communicate within the nodes through multi-hop wireless link to achieve mutual communication. The entire network has no fixed infrastructure, such as base stations. Each node in the network can be used as a router to forward data to other nodes. So it becomes the key of wireless ad hoc network design to develop a dynamic routing protocol that can effectively find the route between nodes. Therefore, this routing protocol should achieve the following functions:

\subsection{Ability of sensing changes in the network topology.}

Routing protocols must ensure that the links in the path have strong connectivity as wireless ad hoc networks require multi-hop communication. A node in a wireless ad hoc network must keep a sense of the neighborhood and the nodes with which it can communicate directly. There are mainly two kinds of methods that the wireless ad hoc network connects the internet: The plane route network structure and the hierarchical route network structure. In a planar routing network structure, all nodes are level and packet routing is based on peer-to-peer connections. However, in a hierarchical routing structure, the lower layer must have at least one node as the gateway to contact with the upper layer.

\subsection{Maintenance of the network topology connection.}

The network topology changes frequently because each node's relative position can be changed at any time. The link state must be dynamically updated and reconfigured in order to maintain strong connectivity between links between nodes.

If the center-controlled routing algorithm is adopted, it is obviously not suitable to transfer too much time and energy to change the link state of the node to all the nodes. So fully distributed intelligent routing algorithm is adopted;

\subsection{Highly adaptive routing.}

Compared with the static nodes in a wired network, wireless ad hoc networks require a highly adaptive routing mechanism to handle rapid topology changes. Traditional routing protocols, such as distance vector and link state algorithms, require a large amount of routing information exchange between designated routers and thus can not effectively work in a wireless ad hoc network. So, a new routing algorithm is proposed for the characteristics of wireless ad hoc networks. Summarily, these routing algorithms fall into three categories: table-driven, demand-driven, and mixed-driven algorithm. Table-driven routing protocol uses periodic routing packet broadcasting to exchange routing information, and each node maintains the routes to all nodes in the entire network. The 
demand-driven routing protocol is based on the needs of sending nodes and performs the discovery process as needed. The network topology structure and routing table content are also built based on demand, so its contents may simply be part of the overall network topology information. The router protocols classification, as shown in the Fig. 1

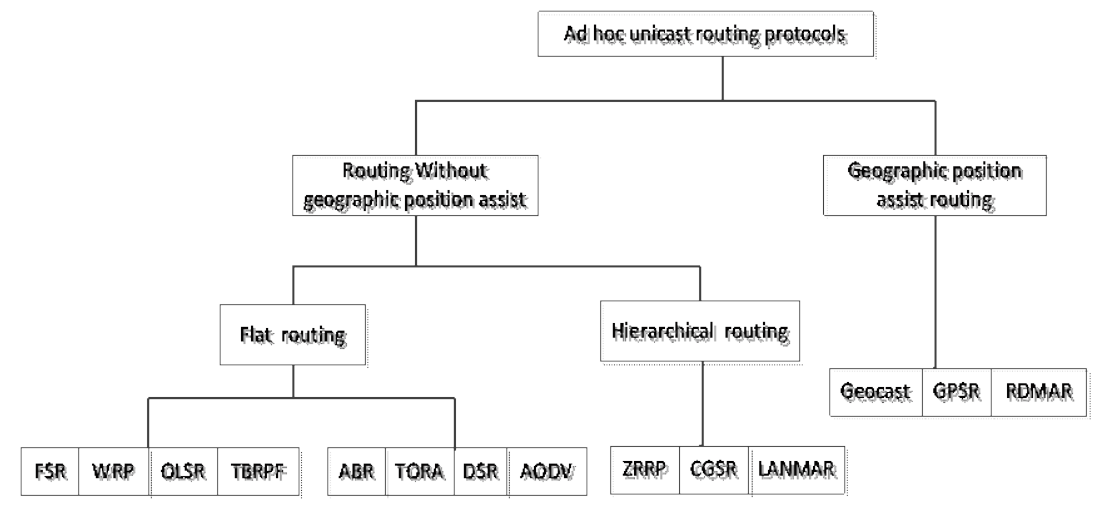

Fig. 1 The main types of routing protocols

\section{Time frame design}

Reasonable time frame design is an important part of the system performance. In order to meet the complicated data transmission requirements and the distributed TDMA method of UAV self-organizing network, this section gives a general design of the time frame structure of the system. The communication networking mode of UAV self-organizing network is a cluster-based structural system. The system runs the algorithm called clustering on the first run, and then runs periodically in the round. SYNCg-Positioning, Network Management, Distributed Scheduling, and data transmission will be included in each period. The structure of the time frame of each round is as shown in the figure.

\section{Conclusions}

Based on a multi-dimensional research on UAV self-organizing network, this paper put forward a new structure and management methods and carried out a detailed design to facilitate the development of UAVs cluster, intelligence and network.

\section{References}

[1] Stefano Basagni, Irnrieh Chlamtae, Violet R. Syrotiuk. A Distance Routing Effect Algorithm for Mobility. International Conference on Mobile Computing and Networking Proceedings of the 4th annual ACM/IEEE international conference on Mobile computing and networking. Dallas, Texas, United States.pp:76\}84, 1998.

[2] C. R. Lin and M. Gerla. Adaptive Clustering for MobileWireless Networks. IEEE Journal of Selected Areas on Communications, 15(7):1265-1275, 1997.

[3] Cai Z, Lu M, Wang X. Channel Access-Based Self-Organized Clustering in Ad Hoc Networks [J]. IEEE Trans Mobile Comp, 2003, 2(2):102-113.

[4] C. E. Perkins and P. Bhagwat. Highly Dynamic Destination-Sequenced Distance-Vector Routing (DSDV) for Mobile Computers. In SIGCOMM, pages 234-244, 1994.

[5] D. L. Gu, H., X. Hong, M. Gerla, G Pei, and Y Lee. C-ICAMA, A Centralized Intelligent Channel Assigned Multiple Access for Multi-layer Ad-Hoc Wireless Networks with UAVs. In IEEE WCNC_ 2000.

[6] F R Hainsworth. Precision andnamics of positioning by Canada geese flying in formation. J of Experimental Biology, 1987, vol. 128:445462. 
[7] Weimerskirch H, Martin J, Clerquin Y, et al. Energy saving in flight formation. Nature .2001, 413:697698.

[8] DORIGO M, MANIEZZO V, COLORNI A. The ant system: optimization by a colony of cooperating agents [J]. IEEE Transactions on Systems, Man\&Cybernetics B, 1996, 26 (2):29241.

[9] S.H.Pruett, GJ.Slutz, J.L.Paunicka, et al. Hardware-in-the-loop simulation using open control platform. AIAA-2003-5759. AIAA Modeling\&Simulation Technologies Conference. August 2003, Austin, Texas.

[10] L.Pollini, M.Innocenti, R.Mati. Vision algorithms for formation flight and aerial refueling with optimal marker labeling. AIAA-2005-6010. AIAA Modeling and Simulation Technologies Conference and Exhibit, San Francisco, California, Aug. 15-18, 2005. 